\title{
INVESTIGAÇÃO NA FORMAÇÃO DE PROFISSIONAIS DE EDUCA- ÇÃO FÍSICA E PSICOLOGIA REVELA DÉFICIT NO CONHECIMENTO SOBRE ESTEROIDES ANABÓLICO ANDROGÊNICOS
}

\author{
Vinicius Dias Wilson \\ Universidade Federal Dos Vales Do Jequitinhonha E Mucuri, Diamantina, Minas Gerais, Brasil. \\ Felipe Brandão Bastos \\ Centro Universitario Estacio de Belo Horizonte, Belo Horizonte, Minas Gerais, Brasil. \\ Luiz Henrique de Lisboa Oliveira \\ Centro Universitario Estacio de Belo Horizonte, Belo Horizonte, Minas Gerais, Brasil. \\ André Gustavo Pereira Andrade \\ Universidade Federal de Minas Gerais, Belo Horizonte, Minas Gerais, Brasil.
}

\section{Resumo}

Praticantes de exercícios físicos e atletas têm abusado de esteroides anabólico androgênicos (EAA's), sujeitando-se a um elevado risco de surgimento de reações adversas à saúde física e psíquica. Profissionais de saúde têm o dever de informar a população sobre os riscos da utilização de EAA's. Este estudo investigou a formação de estudantes dos cursos de Psicologia e Educação Física da cidade de Belo Horizonte sobre esse tema analisando-se um questionário respondido por 414 voluntários. Os resultados revelaram que, na formação desses estudantes, pouca atenção tem sido dada quanto ao uso de EAA's. Conclui-se que os estudantes de ambos os cursos não obtiveram conhecimento satisfatório acerca dos EAA's e suas aplicações.

Palavras-chave: Esteroides. Anabolizantes. Educação Física e Treinamento. Psicologia.

\section{RESEARCH ON THE FORMATION OF PHYSICAL EDUCATION AND PSY- CHOLOGY PROFESSIONALS REVEALS DEFICITS IN KNOWLEDGE ABOUT ANABOLIC ANDROGENIC STEROIDS}

\begin{abstract}
Physical exercise practitioners and athletes have abused of anabolic androgenic steroids (EAA's) subject themselves to the occurrence of harmful reactions to physical and mental health. Healthcare professionals should inform people about issues arising from the use of AAS. The present study investigated the professional formation of students of Psychology and Physical Education of the city of Belo Horizonte regarding this topic. Four hundred and fourteen volunteers answered a questionnaire. The results reveal that minor attention has been given to this matter in the formation of these students, resulting in a trivial understanding about the EAA's. It was concluded that these students of EF and PSI did not obtain satisfactory knowledge about the AAS and its applications.
\end{abstract}

Keywords: Steroids. Anabolic Agents. Physical Education and Training. Psychology. 


\title{
INVESTIGACIÓN EN LA FORMACIÓN DE LOS PROFESIONALES DE LA EDU- CACIÓN FÍSICA Y PSICOLOGÍA REVELA DÉFICIT EN EL CONOCIMIENTO ACERCA DE LOS ESTEROIDES ANABÓLICOS ANDROGÉNICOS
}

\begin{abstract}
Resumen
Los practicantes de ejercicio físico y atletas han abusado de los esteroides anabólicos androgénicos (EAA's), sujetándose a un alto riesgo de surgimiento de reacciones adversas a la salud física y psíquica. Los profesionales de la salud deben informar a la población sobre los riesgos de la utilización de EAA's. Este estudio investigó la formación de los estudiantes de Psicología y Educación Física de la ciudad de Belo Horizonte, con respecto a este tema. El instrumento investigativo fue el análisis de un cuestionario respondido por 414 voluntarios. Los resultados revelaron que poca atención acerca de los EAA's y de sus aplicaciones se ha dado en la formación de estos estudiantes. Se concluyó que los estudiantes de ambos cursos no obtuvieron conocimiento satisfactorio.
\end{abstract}

Palabras clave: Esteroides. Anabolizantes. Educación y Entrenamiento Físico. Psicología.

\section{Introdução}

Os esteroides anabólicos androgênicos (EAA's) são hormônios naturais ou sintéticos derivados da testosterona, sendo usados com fins terapêuticos (KUHN, 2002) desde 1935 (GRAPHERY, 1995). Todavia, em décadas recentes, o seu uso não terapêutico tem se difundido entre jovens (DOMINGUES, S. F.; MARINS, 2007; DURANT; ESCOBEDO; HEATH, 1995; FERREIRA et al., 2007; SCOTT; WAGNER; BARLOW, 1996), adultos (FORTUNATO; ROSENTHAL; CARVALHO, 2007; FRIZON; MACEDO; YONAMINE, 2005; KANAYAMA et al., 2010) e praticantes de exercícios físicos (CUNHA et al., 2004; GRAPHERY, 1995; GRUBER; POPE JR, 2000; HANDELSMAN; GUPTA, 1997; HILDEBRANDT; LANGENBUCHER; G, 2004; IRIART, JORGE ALBERTO BERNSTEIN; ANDRADE, 2002; KANAYAMA et al., 2006, 2010; KUHN, 2002; POPE; KATZ, 1990). Mesmo que as hospitalizações devido ao elevado consumo não terapêutico sejam relativamente raras (JUNIOR; DA, 2013), esses usuários estão mais suscetíveis às reações adversas dos EAA's. Estudos têm observado que o aumento desse uso indevido tem-se tornado um emergente problema de saúde pública (CONWAY et al., 2000; CUNHA et al., 2004).

Assim sendo, recomenda-se que profissionais da saúde tenham interesse e conhecimento por este assunto, pois podem auxiliar usuários e ex-usuários na interrupção do uso (DURANT; ESCOBEDO; HEATH, 1995; GRAPHERY, 1995; KANAYAMA et al., 2010; NOGUEIRA et al., 2015). No tocante ao educador físico, um estudo de 2007 demonstrou que a maioria dos praticantes de musculação buscavam informações sobre os EAA's através desses profissionais, sendo apontados como a fonte predileta de informação e orientação antes mesmo da internet, revistas e jornais científicos e a classe médica (DOMINGUES; MARINS, 2007). Segundo as diretrizes curriculares nacionais do curso de graduação em Educação Física, estabelecidas no parecer número 0138/2002 do Conselho Nacional de Educação/Câmara de Educação Superior (CNE/CES), os currículos propostos pelas instituições de ensino devem fornecer conteúdos que construam um perfil acadêmico e profissional capaz de prestar serviços à sociedade, disseminando e aplicando conhecimentos atualizados que resultem em benefício à saúde da população (MACEDO et al., 2002). Essas responsabilidades podem ser observadas na sentença registrada no primeiro parágrafo da página 4 deste parecer:

Atenção à saúde: como profissional da área de saúde, dentro do âmbito da Educação Física, deve estar apto a desenvolver ações de prevenção, reabilitação, promoção e proteção da saúde, tanto em nível individual quanto coletivo. O profissional de Edu- 
cação Física deve assegurar que sua prática seja realizada de forma segura, integrada e contínua com as demais instâncias do sistema de saúde. (MACEDO et al., 2002).

Ainda sobre as competências do profissional de Educação Física, o parecer define que o profissional deve promover estilos de vida saudáveis, desenvolvendo e aplicando métodos de ensino e atuando em políticas e programas de educação, segurança e vigilância sanitária visando à promoção da saúde em âmbito local, regional e nacional. Fundamentada nessas competências, a matriz curricular das instituições deve conter disciplinas que permitam abordar temas atuais e de relevância social, como os EAA's.

No entanto, um estudo realizado em 2017 encontrou uma prevalência de 9,8\% de usuários de EAA's dentre uma população de 4860 praticantes de exercícios em academia, onde os amigos eram a maior fonte de obtenção de conhecimento a respeito destas substâncias e os professores eram a maior fonte de obtenção das próprias substâncias (ALTHOBITI et al., 2018). Apesar deste recente estudo, existe uma escassez de estudos abordando o uso não terapêutico de EAA's e a carência de dados epidemiológicos indicando a extensão do consumo dessas substâncias contribui para o aumento desse problema de saúde pública (KANAYAMA et al., 2006; LISE et al., 1999; MAYCOCK; HOWAT, 2005; MIDDLEMAN; DURANT, 1996), sugerindo falta de conhecimento dos profissionais de saúde, o que ressalta a importância de se abordar os EAA's na grade curricular durante a graduação desses profissionais.

Apesar de um estudo ter demonstrado que 36,7\% dos estudantes de Educação Física (EF) utilizavam EAA's (ABRAHIN et al., 2013), treinadores esportivos e preparadores físicos devem estar cientes dos riscos envolvidos com o uso de EAA's para poderem aconselhar possíveis usuários (DURANT; ESCOBEDO; HEATH, 1995; MIDDLEMAN; DURANT, 1996; WU, 1997). O uso também está associado a outros tipos de comportamentos de riscos, tais como: o compartilhamento de seringas (FILHO et al., 2011; IRIART, JORGE ALBERTO BERNSTEIN; ANDRADE, 2002); abuso de álcool e drogas ilícitas, como maconha e cocaína; tabagismo e hormônio de crescimento (ALTHOBITI et al., 2018); direção veicular perigosa (DURANT; ESCOBEDO; HEATH, 1995; NILSSON et al., 2001); comportamento agressivo (NILSSON et al., 2001; SCOTT; WAGNER; BARLOW, 1996); possibilidade de desenvolvimento de dependência psíquica e distúrbios de imagem corporal (HILDEBRANDT; LANGENBUCHER; G, 2004; POPE; KATZ, 1990; QUAGLIO et al., 2009), uma vez que observa-se entre estudantes de EF a importância de padrões físicos musculosos (CAMPANA et al., 2014). Consequentemente a esse quadro epidêmico, este estudo foi realizado com o intuito de investigar aspectos relacionados à formação de profissionais de EF e Psicologia (PSI) sobre EAA's.

\section{Metodologia}

Esse estudo apresenta caráter descritivo, quantitativo e qualitativo, realizado com uma amostra intencional não probabilística de 414 alunos do ensino superior cursando o primeiro (PP) e último período (UP) em instituições de ensino superior privadas e públicas da região metropolitana de Belo Horizonte, Minas Gerais. A amostra foi composta de 200 alunos do curso de EF (bacharelado e licenciatura), sendo PP $n=128$ e UP $n=72$ e 214 alunos do curso de PSI, sendo PP n=154 e UP n=60. Um questionário semiestruturado, contendo oito questões objetivas e discursivas, foi elaborado para essa pesquisa. As questões de múltipla escolha permitiam ao avaliado escolher, se necessário, mais de uma opção para a mesma pergunta. A análise dos dados teve como objetivo a investigação grupal e não individual. Voluntários com idade inferior a 18 anos foram excluídos da amostra.

O estudo foi submetido e aprovado pelo Comitê de Ética em Pesquisa do Hospital Sofia Feldman (CAAE: 0024.0.439.000-11), além de estar conforme as Resoluções n: 466/2012 e nº: 510/2016, do Conselho Nacional de Saúde. A autorização para aplicação dos questioná- 
rios nas instituições foi obtida junto à coordenação de cada curso através de cartas de anuência. Após lerem e assinarem um termo de consentimento livre e esclarecido, os voluntários responderam individualmente ao questionário, sem consulta.

Na questão 01 do questionário, com o objetivo de classificar a resposta como satisfatória (SIM) ou insatisfatória (NÃO), a resposta de cada participante da pesquisa foi comparada com definições de EAA's de três estudos, (DURANT; ESCOBEDO; HEATH, 1995; MIDDLEMAN; DURANT, 1996; WU, 1997).

\section{Análise Estatística}

A análise estatística comparou a frequência de respostas entre os cursos de EF e PSI, bem como entre a de PP e UP dentro de cada curso. Foi realizado o teste qui-quadrado $\left(\mathrm{x}^{2}\right)$ de proporções, com nível de significância adotado de $\mathrm{p}<0,05$. O teste compara a frequência observada com a esperada (valor esperado $=$ VE) e, de acordo com Callegari-Jacques (2008, p.139), se o resíduo ajustado for maior, em módulo, a 1,96, a diferença entre eles é considerada significativa. A medida de $V$ de $\operatorname{Cramer}\left(V=\frac{\sqrt{x^{2}}}{\sqrt{N(m-1)}}\right.$, onde $\mathrm{N}=$ total de respostas e $\mathrm{m}=$ número mínimo de colunas ou linhas) foi utilizada para verificar o tamanho do efeito, sendo que para um $\mathrm{df}=3$ (grau de liberdade), $V=0,06$ corresponde a um pequeno efeito de associação; $V=0,17$ a um efeito médio e $V=0,29$ a um efeito grande (COHEN, 1988). O pacote estatístico utilizado foi o SPSS 18.0. Em algumas das questões, a análise estatística foi realizada com base nas respostas quantitativas, qualitativas ou em ambas as respostas; para as respostas quantitativas, considerou-se o número de indivíduos que assinalaram SIM ou NÃO como resposta. Porém, nas respostas qualitativas, considerou-se o número de indivíduos que foram ou não capazes de descrever corretamente algo que sustentasse a opção assinalada anteriormente. A Figura 1 representa os valores percentuais das respostas da questão 08, analisadas através dos valores absolutos das respostas de cada grupo.

\section{Resultados}

Na primeira questão, "Descreva em linhas gerais o que você entende por EAA", observou-se que o número de respostas de EF/UP/SIM foi significativamente maior que o $\mathrm{EF} / \mathrm{PP} / \mathrm{SIM}$, entretanto, o valor de PSI/PP/SIM foi significativamente maior que PSI/UP/SIM. Todos esses grupos apresentaram valores inferiores de respostas satisfatórias em relação ao VE. Não houve diferença significativa quando realizada a comparação entre cursos. Todos os resultados das questões (01 a 07) estão descritos na Tabela 1.

Na questão 02, "Você conhece algum tipo de EAA?", os resultados entre períodos demonstraram um número de respostas do EF/PP/SIM e PSI/PP/SIM significativamente maior que o EF/UP/SIM e PSI/UP/SIM, respectivamente. Ambos EF/PP/SIM e EF/UP/SIM que responderam satisfatoriamente (SIM) apresentaram valores superiores ao VE. O número de respostas EF/PP/NÃO foi significativamente maior que EF/UP/NÃO. O número de respostas PSI/PP/NÃO também foi significativamente maior que PSI/UP/NÃO. Os grupos $\mathrm{PSI} / \mathrm{PP} / \mathrm{NÃO}$ e PSI/UP/NÃO forneceram valores superiores ao respectivo VE.

Porém, ao comparar os cursos nessa questão, obteve-se os seguintes resultados: o número de respostas de EF/PP/SIM foi significativamente maior em relação ao PSI/PP/SIM e apenas o EF/PP/SIM apresentou valor superior ao $\mathrm{VE}^{1}$; o número de respostas de EF/UP/SIM foi significativamente maior que PSI/UP/SIM e apenas o EF/UP/SIM foi superior ao $\mathrm{VE}^{1}$; o número de respostas EF/PP/NÃO foi significativamente inferior que PSI/PP/NÃO, sendo que apenas o PSI/PP/NÃO foi superior ao $\mathrm{VE}^{1}$; não houve diferença significativa entre EF/UP/NÃO e PSI/UP/NÃO e apenas o PSI/UP/NÃO apresentou um valor superior ao VE ${ }^{1}$. 
A comparação entre os períodos das respostas quantitativas dadas a questão 03, "Você conhece algum efeito positivo ou benéfico à saúde relacionado ao uso de EAA? Quais?", demonstrou os seguintes resultados: o número de respostas de EF/PP/SIM e PSI/PP/SIM foram significativamente maiores que EF/UP/SIM e PSI/UP/SIM, respectivamente; todos os grupos apresentaram valores de respostas afirmativas menores ou iguais aos respectivos VE's, apesar do número de respostas de EF/PP/NÃO e PSI/PP/NÃO terem sido significativamente maiores que EF/UP/NÃO e PSI/UP/NÃO, respectivamente. A quantidade de respostas negativas apresentada pelo EF/PP/NÃO e PSI/PP/NÃO foram superiores ao VE.

Entre os períodos, nas respostas qualitativas da questão 03, pode-se obter os seguintes resultados: as respostas de EF/UP/SIM foram significativamente maiores em relação ao $\mathrm{EF} / \mathrm{PP} / \mathrm{SIM}$; o número de respostas de PSI/PP/SIM foi significativamente maior que PSI/UP/SIM; todos os grupos apresentaram valores de respostas afirmativas menores aos respectivos VE; o número de respostas de EF/PP/NÃO e PSI/PP/NÃO foram significativamente maiores que EF/UP/NÃO e PSI/UP/NÃO, respectivamente e somente o valor de $\mathrm{PSI} / \mathrm{UP} / \mathrm{N} \tilde{\mathrm{A} O}$ foi inferior ao VE.

A análise estatística das respostas quantitativas da questão 04 na comparação dos cursos resultou em um número de respostas de PSI/PP/SIM significativamente maior que o EF/PP/SIM, além de somente o PSI/PP/SIM apresentar um valor maior do que o $\mathrm{VE}^{1}$. Não houve diferença significativa entre PSI/UP/SIM e EF/UP/SIM, e apenas o PSI/UP/SIM apresentou um valor maior que o $\mathrm{VE}^{1}$. Também não houve diferença significativa entre EF/PP/NÃO e PSI/PP/NÃO, e apenas o EF/PP/NÃO apresentou um valor maior que o VE. Não houve diferença significativa entre EF/UP/NÃO e PSI/UP/NÃO, e apenas PSI/UP/NÃO apresentou um valor menor que o $\mathrm{VE}^{1}$.

Para análise dos resultados a respeito do uso atual ou pregresso de EAA's, foi feita a comparação entre períodos através das respostas da questão 05, "Você já utilizou ou utiliza algum tipo de EAA?". Apesar da similaridade dos VE's, houve diferença significativa entre o número de respostas de EF/PP/SIM e EF/UP/SIM, e ambos apresentaram valores menores que o VE. O número de respostas de PSI/PP/SIM foi significativamente maior que PSI/UP/SIM, e ambos apresentaram valores maiores que o VE. O número de respostas de EF/PP/NÃO e PSI/PP/NÃO foi significativamente maior que EF/UP/NÃO e PSI/UP/NÃO respectivamente; entretanto apenas PSI/UP/NÃO apresentou um valor menor que o VE.

Quanto à comparação entre os cursos dessa questão 05 , o número de respostas apresentado por EF/PP/SIM foi significativamente superior a PSI/PP/SIM, e apenas EF/PP/SIM apresentou um valor maior que o $\mathrm{VE}^{1}$. Não houve diferença significativa entre EF/UP/SIM e $\mathrm{PSI} / \mathrm{UP} / \mathrm{SIM}$, apenas o EF/UP/SIM teve um valor que alcançou o $\mathrm{VE}^{1}$. O número de respostas de PSI/PP/NÃO foi significativamente maior que EF/PP/NẪO, e apenas o PSI/PP/NÃO apresentou um valor maior que o $\mathrm{VE}^{1}$. O número de respostas de PSI/UP/NÃO foi significativamente maior que EF/UP/NÃO, e apenas PSI/UP/NÃO apresentou um valor maior que o VE" ${ }^{1}$.

Os resultados da questão 06, "Você conhece alguém que utiliza ou utilizou EAA?", foram utilizados apenas para comparar os cursos; eles não apresentaram diferença significativa entre EF/PP/SIM e PSI/PP/SIM e apenas esse último apresentou um valor maior que o $\mathrm{VE}^{1}$. O número de respostas de EF/UP/SIM foi significativamente superior ao de PSI/UP/SIM; apenas o primeiro teve um valor maior que $\mathrm{VE}^{1}$. O número de respostas de PSI/PP/NÃO e $\mathrm{PSI} / \mathrm{UP} / \mathrm{NÃO}$ foi significativamente maior que EF/PP/NÃO e EF/UP/NÃO respectivamente; e ambos, PSI/PP/NÃ̃ e PSI/UP/NÃO, apresentaram valores acima do VE ${ }^{1}$.

Para levantamento de dados a respeito da opinião dos participantes sobre o uso não terapêutico de EAA, foi feita a comparação entre períodos através das respostas dadas à questão 07, “Você concorda com uso de EAA's por fins não terapêuticos ou profiláticos?". Conforme mostra a Tabela 1, o número de respostas de EF/PP/SIM e PSI/PP/SIM foram significativamente superiores aos de EF/UP/SIM e PSI/UP/SIM respectivamente; todos apresentaram va- 
lores inferiores ao VE; o número de respostas de EF/PP/NÃO e PSI/PP/NÃO foram significativamente maiores que EF/UP/NÃO e PSI/UP/NÃO respectivamente e apenas o PSI/UP/NÃO apresentou um valor inferior ao VE.

Ao confrontar os cursos, as repostas da questão 07 apresentaram uma ausência de diferença significativa entre EF/PP/SIM vs. PSI/PP/SIM e EF/UP/SIM vs. PSI/UP/SIM, e os grupos PSI/PP/SIM e EF/UP/SIM apresentaram valores maiores que o $\mathrm{VE}^{1}$. O número de respostas de PSI/PP/NÃO foi significativamente maior que EF/PP/NÃO, e apenas o PSI/PP/NÃO apresentou um valor maior que o $\mathrm{VE}^{1}$. Não houve diferença significativa entre EF/UP/NÃO e $\mathrm{PSI} / \mathrm{UP} / \mathrm{NÃO}$ e o primeiro apresentou um valor maior que o $\mathrm{VE}^{1}$.

Os dois cursos, EF e PSI, no que concerne o tamanho do efeito ( $V$ de Cramer), apresentaram um grande efeito para todas as questões, à exceção do curso EF na questão 02 e de ambos os cursos na questão 06, onde o efeito foi médio (Tabela 1): 
Tabela 1 - Valores das respostas das questões 01 à 07 , classificadas por cursos e períodos.

\begin{tabular}{|c|c|c|c|c|c|c|c|}
\hline Questões $\quad$ Grupos & & PP/SIM & UP/SIM & PP/NÃO & UP/NÃO & VE & $\begin{array}{c}\mathrm{V} \\
\text { Cramer }\end{array}$ \\
\hline \multirow{5}{*}{$\begin{array}{l}\text { 01) Descreva em linhas gerais ou que } \\
\text { você entende por EAA. }\end{array}$} & EF & $1^{*}$ & 2 & $128^{*}$ & 69 & 66,7 & 0,61 \\
\hline & r.a. & 6,87 & 6,75 & 18,81 & 3,53 & & \\
\hline & PSI & $2 *$ & 1 & $152^{*}$ & 59 & 53,5 & 0,67 \\
\hline & r.a. & 7,02 & 7,47 & 16,09 & 0,87 & & \\
\hline & $\mathrm{VE}^{1}$ & 1,5 & 1,5 & 140 & 64 & & \\
\hline \multirow{5}{*}{ 02) Você conhece algum tipo de EAA? } & $\mathbf{E F}$ & $53 * \S$ & $31^{\$}$ & $71^{* *}$ & 36 & 47,8 & 0,19 \\
\hline & r.a. & 0,67 & 2,81 & 3,97 & 2,06 & & \\
\hline & PSI & $29^{*}$ & 9 & $122 *$ & 51 & 52,8 & 0,47 \\
\hline & r.a. & 3,53 & 6,16 & 14,66 & 0,28 & & \\
\hline & $\mathrm{VE}^{1}$ & 41 & 20 & 96,5 & 43,5 & & \\
\hline \multirow{5}{*}{$\begin{array}{l}\text { 03) Você conhece algum efeito positivo } \\
\text { ou benéfico à saúde relacionado ao } \\
\text { uso de EAA? Quais? }\end{array}$} & EF & $25^{*}$ & 18 & $98^{*}$ & 43 & 46,6 & 0,39 \\
\hline & r.a. & 03,69 & 4,66 & 10,24 & 0,98 & & \\
\hline & PSI & $27^{*}$ & 18 & $125^{*}$ & 41 & 52,8 & 0,47 \\
\hline & r.a. & 3,8 & 5,01 & 15,56 & 1,81 & & \\
\hline & $\mathrm{VE}^{1}$ & 26 & 18 & 111,5 & 42 & & \\
\hline \multirow{5}{*}{$\begin{array}{l}\text { 03) Você conhece algum efeito positivo } \\
\text { ou benéfico à saúde relacionado ao } \\
\text { uso de EAA? Quais? (A. Qualitativa) }\end{array}$} & EF & $20^{* 8}$ & $21^{\$}$ & $108^{* \#}$ & $51^{f}$ & 50 & 0,41 \\
\hline & r.a. & 4,37 & 4,23 & 12,43 & 0,33 & & \\
\hline & PSI & $25^{*}$ & 17 & $129^{*}$ & 43 & 53,5 & 0,48 \\
\hline & r.a. & 4,07 & 5,13 & 16,64 & 1,51 & & \\
\hline & $\mathrm{VE}^{1}$ & 22,5 & 19 & 118,5 & 47 & & \\
\hline \multirow{5}{*}{$\begin{array}{l}\text { 04) Você conhece algum efeito } \\
\text { negativo ou risco à saúde relacionado } \\
\text { ao uso de EAA? }\end{array}$} & EF & $87 * \$$ & 41 & $30^{*}$ & 9 & 41,8 & 0,4 \\
\hline & r.a. & 7,84 & 1,32 & 3 & 5,87 & & \\
\hline & PSI & $124^{*}$ & 51 & $22 *$ & 8 & 51,3 & 0,51 \\
\hline & r.a. & 15,59 & 0,29 & 4,49 & 6,29 & & \\
\hline & $\mathrm{VE}^{1}$ & 105 & 46 & 26 & 8,5 & & \\
\hline \multirow{5}{*}{$\begin{array}{l}\text { 05) Você já utilizou ou utiliza algum } \\
\text { tipo de EAA? }\end{array}$} & EF & $10^{* \S}$ & 10 & $111^{* \#}$ & $44^{f}$ & 43,8 & 0,55 \\
\hline & r.a. & 5,74 & 5,74 & 14,65 & 0,83 & & \\
\hline & PSI & $2^{*}$ & 0 & $153^{*}$ & 58 & 71 & 0,51 \\
\hline & r.a. & 7,02 & 7,27 & 25,98 & 0,84 & & \\
\hline & $\mathrm{VE}^{1}$ & 6 & 10 & 132 & 51 & & \\
\hline \multirow{5}{*}{$\begin{array}{l}\text { 06) Você conhece ou conheceu alguém } \\
\text { que utiliza ou utilizou EAA? }\end{array}$} & EF & 79 & $56^{5}$ & $41^{* \#}$ & $16^{f}$ & 48 & 0,28 \\
\hline & r.a. & 5,59 & 1,19 & 1,29 & 4,92 & & \\
\hline & PSI & 85 & 28 & $68^{*}$ & 29 & 52,5 & 0,27 \\
\hline & r.a. & 5,74 & 3,67 & 2,54 & 3,53 & & \\
\hline & $\mathrm{VE}^{1}$ & 82 & 42 & 54,5 & 22,5 & & \\
\hline \multirow{5}{*}{$\begin{array}{l}\text { 07) Você concorda com a utilização de } \\
\text { EAA por fins não terapêuticos ou } \\
\text { profiláticos? }\end{array}$} & EF & $22 *$ & 20 & $84^{* \#}$ & 49 & 43,8 & 0,34 \\
\hline & r.a. & 4,13 & 4,4 & 6,93 & 0 & & \\
\hline & PSI & $23^{*}$ & 14 & $115^{*}$ & 36 & 47 & 0,49 \\
\hline & r.a. & 4,38 & 5,55 & 13,74 & 2,57 & & \\
\hline & $\mathrm{VE}^{1}$ & 22,5 & 17 & 99,5 & 42,5 & & \\
\hline
\end{tabular}

Valores representam respostas afirmativas = SIM e negativas $=$ NÃO; porém apenas na questão 01, SIM = respostas satisfatórias e $\mathrm{NÃO}=$ não-satisfatórias. EF=Educação Física; $\mathrm{PSI}=$ Psicologia; $\mathrm{PP}=$ Primeiro período; UP=Ultimo período; r.a.= Resíduo ajustado; $\mathrm{VE}=$ Valor Esperado entre os periodos do mesmo curso e $\mathrm{VE}{ }^{1}=$ Valor Esperado entre os cursos. ${ }^{*}$ Diferença significativa do $\mathrm{PP} / \mathrm{SIM}$ em relação ao UP/SIM e do PP/NÃO em relação ao UP/NÃO para cada curso. ${ }^{\S}$ Diferença significativa do EF/PP/SIM em relação ao PSI/PP/SIM. "Diferença significativa do EF/UP/SIM em relação ao PSI/UP/SIM. "Diferença significativa do EF/PP/NÃO em relação ao PSI/PP/NÃO. ${ }^{f}$ Diferença significativa do Grupo EF/UP/NÃO em relação ao PSI/UP/NÃO. Os grupos foram comparados usando o teste qui-quadrado de proporções, com nivel de significância adotado de p $<0,05$, teste que determinou o VE.

$\mathrm{Na}$ questão 08 , foi solicitado aos voluntários que apontassem as principais fontes de informações a respeito dos EAA's. Os resultados obtidos, expressos na Figura 1(A), apontam que os meios de comunicação em massa ( $\mathrm{MC}=$ mídia através de televisão, rádio, jornal impresso e internet) e o senso comum ( $\mathrm{SC}=$ conhecimento empírico) são os principais meios de obtenção de informações utilizados pelos grupos no PP. No entanto, foi observado um aumento da contribuição do conteúdo curricular (CC) em ambos os cursos, porém houve uma diminuição significativa do MC somente no curso EF. Todos os percentuais de respostas do grupo 
EF/UP foram significativamente diferentes do PSI/UP. Ao observar a Figura 1(B), verificouse que as diferenças $($ delta $=\Delta$ ) entre os percentuais do UP e PP no curso de EF foram significativamente diferentes ao PSI para: MC, AA, CC e OU, derivando em deltas mais positivos nas $\mathrm{AA}$ e $\mathrm{CC}$ em prol de deltas mais negativos nos $\mathrm{MC}$ e outras fontes. $\mathrm{O} \Delta$ do $\mathrm{SC}$ não apresentou diferença.

\section{Figura 1}
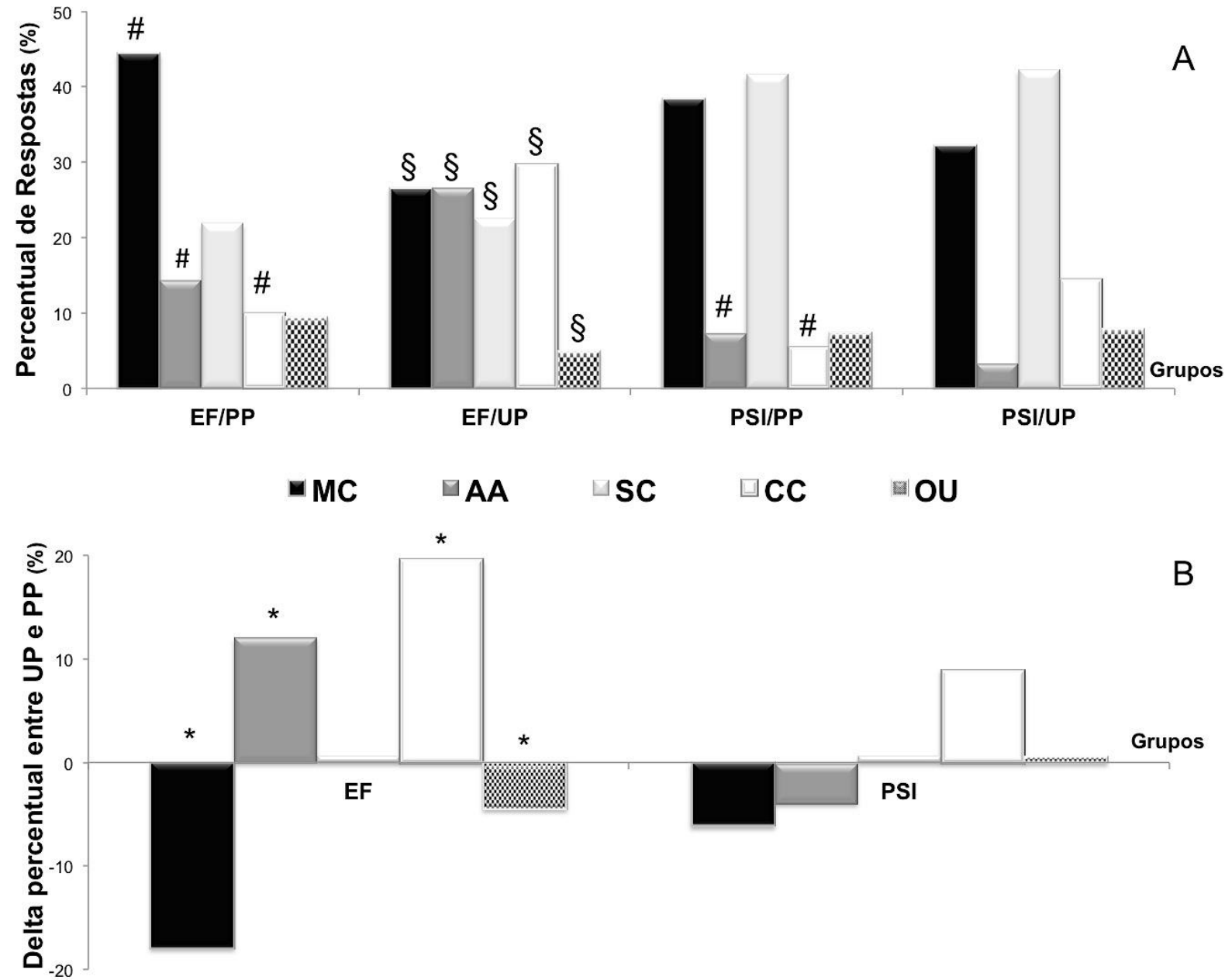

Fontes de informação sobre EAA's apontadas pelos estudantes. "Diferença significativa em relação ao UP do respectivo curso; ${ }^{\S}$ diferença significativa em relação ao mesmo período do curso PSI; *Diferença significativa em relação ao curso PSI. $\mathrm{EF}=$ Educação Física; PSI=Psicologia; PP=Primeiro período; UP=Último período. EF/PP n=160, EF/UP n=102, PSI/PP $\mathrm{n}=219, \mathrm{PSI} / \mathrm{UP} \mathrm{n}=90 . \mathrm{MC}=$ Meios de comunicação em massa, $\mathrm{AA}=$ Atividades acadêmicas, $\mathrm{SC}=$ Senso Comum, $\mathrm{CC}=$ Conteúdo Curricular, $\mathrm{OU}=$ Outros. $\mathrm{EF} / \mathrm{PP} \mathrm{MC}=44,37 \%, \mathrm{AA}=14,37 \%, \mathrm{SC}=21,87 \%, \mathrm{CC}=10 \%$ e $\mathrm{OU}=9,37 \%$. $\mathrm{EF} / \mathrm{UP}$ $\mathrm{MC}=26,47 \%, \mathrm{AA}=26,47 \%, \mathrm{SC}=22,54 \%, \mathrm{CC}=29,60 \%$ e $\mathrm{OU}=4,9 \% . \mathrm{PSI} / \mathrm{PP} \mathrm{MC}=38,35 \%, \mathrm{AA}=7,3 \%, \mathrm{SC}=41,55 \%$, $\mathrm{CC}=5,47 \%$ e $\mathrm{OU}=7,3 \%$. PSI/UP MC $=32,22 \%, \mathrm{AA}=3,33 \%, \mathrm{SC}=42,22 \%, \mathrm{CC}=14,44 \%$ e OU=7,77\%. Delta $(\Delta)=\mathrm{UP}-\mathrm{PP} ; \mathrm{EF}$ $(\Delta \mathrm{MC}=-17,90 \%, \Delta \mathrm{AA}=12,10 \%, \Delta \mathrm{SC}=0,67 \%, \Delta \mathrm{CC}=19,60 \%, \Delta \mathrm{OU}=-4,47 \%)$ e PSI $(\Delta \mathrm{MC}=-6,13 \%, \Delta \mathrm{AA}=-3,97 \%, \Delta-$ $\mathrm{SC}=0,67 \%, \Delta \mathrm{CC}=8,97 \%, \Delta \mathrm{OU}=0,47 \%)$.

\section{Discussão}

Na questão 01, o número de respostas satisfatórias do EF/UP foi significativamente maior do que o EF/PP, o que indica que a formação em EF pode proporcionar maior entendimento sobre o que são EAA's. A mesma afirmativa não pode ser feita a respeito do curso de PSI, pois o PSI/PP foi significativamente superior no número de respostas satisfatórias dadas a esta questão em comparação ao UP. Embora tenham ocorrido essas diferenças entre períodos, não foi encontrada diferença significativa na comparação entre os cursos. 
Nessa questão, o dado mais relevante é que nenhum dos grupos alcançou o VE de respostas satisfatórias. Hipoteticamente, esperava-se que pelo menos os grupos EF/UP e PSI/UP apresentassem números de respostas superiores ao VE. Os indivíduos destes grupos encontravam-se muito próximos da conclusão de sua graduação e do início do livre exercício de suas respectivas profissões, logo esse resultado aponta para uma possível carência que poderá se apresentar a usuários e ex-usuários de EAA's ao buscarem auxílio profissional. De acordo com Kanayama et al. (2010), o número de usuários e ex-usuários de EAA’s que busca auxílio profissional com o intuito de interromper o uso possivelmente irá aumentar nos próximos anos, visto que os jovens usuários na época da popularização do uso estão agora chegando a meia idade, estando mais propensos à complicações cardíacas e psiconeuroendócrinas decorrentes do uso não terapêutico dessas drogas, o que sugere que profissionais de múltiplas áreas clínicas e aquelas em contato com homens de meia idade devem estar atentos a possibilidade do desenvolvimento da dependência de EAA's, além dos riscos associados ao seu uso, para poder auxiliá-los.

Uma pesquisa realizada com praticantes de musculação no ano de 2007 , na cidade de Belo Horizonte, demonstrou que 51,5\% dos participantes do estudo afirmaram solicitarem a professores de EF informações sobre suplementos alimentares e EAA's, denominados na referida pesquisa como recursos ergogênicos (DOMINGUES; MARINS, 2007) e 85\% dos participantes afirmaram terem utilizado ou conhecerem algum usuário não terapêutico de EAA's. Sendo assim, devido à baixa satisfatoriedade das respostas obtidas na questão 01, surgem dúvidas sobre a real capacidade dos futuros profissionais de EF e PSI de auxiliaram de forma satisfatória e fornecerem informações fidedignas sobre os EAA's aos possíveis usuários e/ou ex-usuários. Considerando que esta realidade indica uma falha no processo de educação, sugere-se que instituições de ensino, órgãos públicos e corpos docentes estejam mais atentos às evidências atuais que apontam para a necessidade de preparação profissional e aumento no entendimento sobre EAA's, visando combater o aumento de seu uso indiscriminado por usuários não terapêuticos (DOMINGUES; MARINS, 2007; DURANT; ESCOBEDO; HEATH, 1995; GRAPHERY, 1995; KANAYAMA et al., 2010; LISE et al., 1999; MIDDLEMAN; DURANT, 1996; WU, 1997).

Os grupos EF/PP e PSI/PP apresentaram na questão 02 uma quantidade de respostas positivas, significativamente superior aos respectivos grupos do UP. Estes dados podem sugerir que o interesse dos futuros profissionais de EF e PSI a respeito de EAA's esteja aumentando. Podem sugerir ainda que, nos indivíduos jovens, houve um aumento da popularidade de tais substâncias, seja pelo uso próprio ou através do contato com usuários.

O curso de EF apresentou maior conhecimento em relação ao curso de PSI (em mesmos períodos), superando o VE das respostas positivas, e o curso PSI para respostas negativas. Deve-se lembrar que durante o período de graduação os estudantes fazem estágio. Caso a prática desses estágios em academias de musculação tenha sido comum no grupo EF, isso pode ter influenciado o aumento dos diferentes tipos de EAA's conhecidos, pois de acordo com estudos em diferentes cidades do Brasil, o consumo de EAA's é bastante difundido nos indivíduos que treinam musculação (ARAUJO; ANDREOLO; SILVA, 2002; DOMINGUES; MARINS, 2007; IRIART; ANDRADE, 2002; SANTOS et al., 2006; SILVA, I. S. M. F.; MOREAU, 2003; SILVA, P. R. P. et al., 2007; VENÂNCIO et al., 2010) e outras atividades físicas (COSTA et al., 2007; FRIZON; MACEDO; YONAMINE, 2005).

As respostas representadas na Figura 1 relativas à oitava pergunta indicam que os MC e SC foram apontados em maior número como as fontes de obtenção de conhecimento a respeito dos EAA's no PP. Sendo assim, é possível que, embora estes estudantes iniciem a sua formação com um maior conhecimento a respeito dos EAA's, esse conhecimento talvez seja obtido, em maior grau, de fontes pouco confiáveis e baixo crivo científico. Clement et al. (2011) citaram que a expansão do acesso à internet pode contribuir para o aumento das infor- 
mações que os jovens obtêm a respeito dos EAA's. Outro estudo realizado nos EUA com o objetivo de investigar o uso da internet por parte dos jovens revelou que $31 \%$ dos participantes afirmaram utilizá-la para buscar informações sobre saúde e aptidão física, e que $22 \%$ afirmaram utilizá-la para informações sobre assuntos que incluíam EAA's (LENHART; MADDEN; HITLIN, 2005). Nesse mesmo ano, uma publicação atentou que o estigma social criado em torno dos EAA's pode contribuir para que os jovens considerem este um assunto difícil de ser tratado (MAYCOCK; HOWAT, 2005). Sendo assim, a internet pode de fato estar servindo como fonte preferencial de informações sobre os EAA's, contrapondo a busca por auxílio de educadores, pais e outras classes profissionais. Mesmo que a pesquisa na internet sobre EAA's seja apenas para finalidade informativa, é provável que isso sirva como fator predisponente para o uso (WICHSTROM, 2006).

Segundo a análise das respostas qualitativas sobre os efeitos positivos ou benéficos à saúde (questão 03), todos os grupos mantiveram valores de respostas satisfatórias abaixo do VE. Os resultados dos grupos do curso EF nas questões 01, 02, 03 e 04 demonstram que o período da graduação foi capaz de proporcionar aumento de conhecimento sobre os benefícios à saúde. Todavia, demonstram que o aumento do conhecimento dos benefícios não atinge um valor satisfatório. A mesma realidade não foi observada no curso PSI. De fato o grupo de PP desse curso demonstrou superioridade em relação ao grupo de UP no que se refere ao conhecimento sobre tais aspectos. Estes resultados, juntamente com os resultados das duas primeiras questões, reforçam a ideia de que a atenção dada a esse assunto ou retenção da informação nos cursos de PSI tem sido possivelmente menor do que no curso de EF. Vale ressaltar que a literatura tem sugerido que profissionais da área de PSI também devem se interessar por esse assunto devido a associação do consumo de EAA's ao desenvolvimento de alterações psíquicas (LISE et al., 1999) e comportamentais (COSTA et al., 2007; GRUBER; POPE JR, 2000; KANAYAMA et al., 2003, 2010; MIDDLEMAN; DURANT, 1996; NILSSON et al., 2001; POPE; KATZ, 1990; SCOTT; WAGNER; BARLOW, 1996; WAGNER, 1989).

A comparação das respostas qualitativas entre os períodos na questão 04 levantou informações referentes ao conhecimento que os indivíduos dos grupos deste estudo têm sobre os efeitos negativos ou prejudiciais à saúde decorrentes do "mau uso" de EAA's. Foi observado que os grupos do PP de ambos os cursos possuem maior conhecimento a respeito dos riscos em relação aos indivíduos do UP de cada curso. Apenas os grupos de PP alcançaram o valor superior de respostas satisfatórias. Na comparação entre cursos, foi encontrada diferença significativa apenas entre o número de respostas satisfatórias dos grupos de PP de ambos os cursos. O grupo PSI/PP demonstrou maior conhecimento a respeito dos efeitos negativos e prejudiciais à saúde. A análise das respostas desta questão reforça os achados das questões 01 , 02 e 03: a abordagem do assunto não produziu resultado no aumento do entendimento desses graduandos sobre EAA's.

Comparando os resultados desta questão 04 com os resultados citados previamente da questão 08, observamos que um número maior de indivíduos do grupo de PSI/PP afirmou obter informações através dos MC e através do SC e assim, pode-se inferir que o tipo de meio de comunicação pode representar diferenças na ênfase que é dada quando se trata dos benefícios ou prejuízos relacionados ao consumo de EAA's.

Em um estudo realizado no Brasil, dez indivíduos que praticavam musculação afirmaram terem sido orientados por educadores físicos sobre o uso de EAA's, o que representou $11 \%$ da amostra total (SILVA, I. S. M. F.; MOREAU, 2003). Contudo, a tendência indicada nesse estudo não foi reforçada pelos dados produzidos na questão 07 do nosso estudo, pois eles indicam que o número de estudantes de ambos os períodos e de ambos os cursos que discordam do uso não terapêutico e profilático é superior ao VE. Nota-se, portanto que, embora estes grupos não tenham conhecimento satisfatório sobre o que são os EAA's, seus benefícios e riscos, aparenta haver entre eles um estigma negativo a respeito do uso não terapêutico. Es- 
ses dados são conflitantes com resultados de estudos já citados, que indicaram que a exposição à informação contida na internet e em MC serve como fator de incentivo ao uso (CLEMENT et al., 2011; WICHSTROM, 2006). Essa divergência pode ser justificada por uma amostragem com formações e bases distintas.

$\mathrm{Na}$ análise dos resultados da questão 05, que investigava a respeito do uso atual ou pregresso de EAA's, observou-se que o grupo de EF/PP/SIM demonstrou superioridade significativa do uso relatado em relação ao grupo PSI/PP/SIM. O percentual de indivíduos participantes neste estudo que afirmou ter feito uso de EAA's foi de 5,71\% nos cursos de EF e de $5,38 \%$ nos cursos de PSI. Um estudo realizado com 382 estudantes universitários de cursos da área da saúde de faculdades públicas no estado de Pernambuco, Brasil, reporta percentuais de uso de $13,0 \%$ para indivíduos do sexo masculino e de 4,5\% para indivíduos do sexo feminino e o percentual total da amostra deste estudo que afirmou ter feito uso de EAA's sem receita médica foi de 7,06\% (COLARES; FRANCA; GONZALEZ, 2009), valor maior do que o observado no presente estudo. Outros estudos realizados com praticantes regulares de exercícios físicos em diferentes regiões do país também obtiveram valores superiores: 20,6\% em João Pessoa - PB (NOGUEIRA et al., 2015), 6,5\% em Erechim e Passo Fundo - RS (FRIZON; MACEDO; YONAMINE, 2005), 11,1\% em Porto Alegre - RS (SILVA, P. R. P. et al., 2007), 19,0\% de usuários e 37,0\% de ex-usuários em Aracaju - SE (SANTOS et al., 2006), 19\% em São Paulo - SP (SILVA, I. S. M. F.; MOREAU, 2003), 9,0\% em Goiânia - GO (ARAUJO; ANDREOLO; SILVA, 2002). Dados bem divergentes aos de outros países: entre frequentadores de academia de ginástica no Reino Unido, 65,8\% a 70\% utilizam ou já utilizaram EAA's (BAKER; GRAHAM; DAVIES, 2006); 3,2\% do sexo masculino e 1,2\% do sexo feminino (HANDELSMAN; GUPTA, 1997) dos estudantes de ensino médio na Austrália; 3,6\% de usuários homens e 2,8\% de mulheres jovens na Suécia (NILSSON et al., 2001).

Apesar da variedade de amostras e resultados, observa-se uma tendência a maiores valores percentuais nos estudos realizados com pessoas fisicamente ativas (ARAUJO; ANDREOLO; SILVA, 2002; DOMINGUES; MARINS, 2007; FRIZON; MACEDO; YONAMINE, 2005; SANTOS et al., 2006; SILVA, I. S. M. F.; MOREAU, 2003; SILVA, P. R. P. et al., 2007), reiterando a responsabilidade profissional do educador físico nesse contexto educacional. Além dos valores percentuais serem menores entre os indivíduos mais jovens, sobretudo naqueles que estão no ensino médio (BAKER; GRAHAM; DAVIES, 2006; HANDELSMAN; GUPTA, 1997; NILSSON et al., 2001). Tanto o presente estudo quanto o estudo com grupos universitários realizado por Colares et al. (2009, p.523) apresentaram valores percentuais de usuários inferiores aos encontrados em praticantes de exercícios físicos e valores superiores aos encontrados entre jovens e estudantes do ensino médio. Esses dados nos levam à hipótese de que a faixa etária e grau de escolaridade também exercem influência sobre a utilização de EAA's.

As respostas da questão 06 indicam que os estudantes do EF/UP têm maior contato com usuários em relação aos do PSI/UP, o que reforça a ideia da possibilidade dos estudantes de EF terem maior contato com usuários de EAA's nos estágios curriculares. Vale ressaltar que nestes períodos os estudantes podem exercer influência positiva no sentido de conscientizarem alunos de academias e de locais de prática de treinamento sobre os possíveis riscos associados ao uso não terapêutico, conforme sugerido por estudos anteriores (DURANT; ESCOBEDO; HEATH, 1995; FRIZON; MACEDO; YONAMINE, 2005; GRAPHERY, 1995; KANAYAMA et al., 2010; LISE et al., 1999; NILSSON et al., 2001). Entretanto, precisam ser preparados e incentivados em sala de aula para que adotem tal conduta. Deve ser ressaltado que, de acordo com Macedo et al. (2002), o educador físico deve estar apto a desenvolver ações de prevenção à saúde, portanto não se pode negligenciar a importância e responsabilidade desse educador, atuando no ensino fundamental e médio para realizar intervenções edu- 
cacionais em crianças e adolescentes antes/durante o ingresso dessas nos centros de treinamentos.

Outros dados ainda indicam uma maior abordagem sobre EAA's durante a formação em EF em relação à PSI: na questão $08,26,47 \%$ dos alunos de EF afirmaram terem aprendido sobre EAA's através de AA, como palestras e seminários, enquanto apenas 3,33\% alunos de PSI fizeram tal afirmativa. Aproximadamente 30\% dos alunos de EF afirmaram terem aprendido sobre EAA's através do CC e apenas 14,44\% de PSI fizeram tal afirmativa. Estes resultados são confirmados ao se observar as respectivas diferenças significativas entre os deltas dos cursos na figura 1B, e podem explicar o que conduziu mais estudantes do UP a não concordarem com uso de tais substâncias (questão 07), expondo a importância de uma abordagem mais frequente durante a formação do profissional.

\section{Conclusão}

Conclui-se que os estudantes de EF e PSI não estão obtendo conhecimento satisfatório a respeito de EAA's e da sua utilização para fins terapêuticos e não terapêuticos durante o período de formação profissional. Todavia, os estudantes de EF evidenciaram maior conhecimento a respeito dos possíveis benefícios quando comparados aos de PSI, e esses, em contrapartida, apresentaram maior conhecimento a respeito dos possíveis malefícios.

Ademais, conclui-se que o maior contato com usuários pelos estudantes de EF em relação aos de PSI não influenciou a opinião do grupo de EF ao ponto de fazê-los indicar o uso dos EAA's. Fica evidente que apesar do curso EF ter obtido melhora nas fontes de informações com conotação científica, o uso de EAA's merece mais atenção em ambos os cursos de graduação. Sugere-se que futuras pesquisas dentro dessa temática poderão investigar outros grupos de estudantes e profissionais, como nutricionistas e médicos.

\section{Referências}

ABRAHIN, O. S. C. et al. Prevalência do uso e conhecimento de esteroides anabolizantes androgênicos por estudantes e professores de educação física que atuam em academias de ginástica. Revista Brasileira de Medicina do Esporte, v. 19, n. 1, p. 27-30, fev. 2013.

ALTHOBITI, S. D.; ALQURASHI, N. M.; ALOTAIBI, A. S.; ALHARTHI, T. F.; ALSWAT, K. A. Prevalence, Attitude, Knowledge, and Practice of Anabolic Androgenic Steroid (AAS) Use Among Gym Participants. Materia Socio-Medica, v. 30, n. 1, p. 49-52, 2018.

ARAUJO, L. R.; ANDREOLO, J.; SILVA, M. S. Utilização de suplemento alimentar e anabolizante por praticantes de musculação nas academias de Goiania - GO. Revista Brasileira de Ciência e Movimento, v. 10, n. 3, 2002.

BAKER, J. S.; GRAHAM, M. S.; DAVIES, B. Steroid and prescription medicine abuse in the health and fitness community: a regional study. European Journal of Internal Medicine, v. 17, n. 7, 2006.

CALLEGARI-JACQUES, S. M. Bioestatística princípios e aplicações. Editora Artmed, 2008. 
CAMPANA, A. N. N. B.; TAVARES, M. DA C. G. C. F. Aspectos da "drive for muscularity" em estudantes de Educação Física. Revista Brasileira de Educação Física e Esporte, v. 28, n. 2, p. 233-248, jun. 2014.

CLEMENT, C. L.; MARLOWE, D. B.; PATAPIS, N. S.; FESTINGER, D. S.; FORMAN, R. F. Nonprescription Steroids On the Internet. Substance Use \& Misuse, v. 1, n. 13, 2011.

COHEN, J. Statistical Power Analysis for the Behavioral Sciences. Routledge, 1988.

COLARES, V.; FRANCA, C. D.; GONZALEZ, E. Condutas de saúde entre universitários: diferenças entre gêneros. Caderno de Saúde Pública, v. 25, n. 3, 2009.

CONWAY, A. J. et al. Use, misuse and abuse os androgens. The endocrine Soceity of Australia consensus guidelines for androgens prescribing. The Medical Journal of Australia, v. 172, n. 5, 2000.

COSTA, S. P. V. E.; GUIDOTO, C.; UZUNIAN, R. F.; VIEBIG, L. G. Distúrbios da imagem corporal e transtornos alimentares em atletas e praticantes de atividade física. Revista Digital, Buenos Aires, ano 12, n. 114, nov. 2007. Disponível em: http: <//www.efdeportes.com/efd114/transtornos-alimentares-em-atletas.htm>. Acesso em: 19 abr. 2017.

CUNHA, T. S. et al. Anabolic androgenic steroids and the relation to the sportive practice. Revista Brasileira de Ciências Farmacêuticas, v. 40, n. 2, p. 165-179, jun. 2004.

DOMINGUES, S.; MARINS, J. C. B. Utilização de recursos ergogênicos e suplementos alimentares por praticantes de musculação em Belo Horizonte - MG. Fit Perf J., v. 6, n. 4, p. 218-226, 2007.

DURANT, R. H.; ESCOBEDO, L. G.; HEATH, G. W. Anabolic-steroid use, strenght traning, and multiple drug use among adolescentes in the united states. Pediatrics, v. 96, n. 1 July, 1995.

FERREIRA, U. M. G. et al. Esteróides anabólicos androgênicos. Revista Brasileira em Promoção de Saúde, v. 20, n. 4, 2007.

FILHO, C. et al. Pyomyositis in athletes after the use of anabolic steroids: case reports. Revista Brasileira de Ortopedia, v. 46, n. 1, p. 97-100, 2011.

FORTUNATO, R. S.; ROSENTHAL, D.; CARVALHO, D. P. Abuso de esteróides anabolizantes e seu impacto sobre a função tireóidea. Arquivo Brasileiro de Endocrionologia e Metabologia, v. 51, n. 9, 2007.

FRIZON, F.; MACEDO, S. M. D.; YONAMINE, M. Uso de esteróides androgenos anabólicos por praticantes de atividade física das principais academias de Erechim e Passo Fundo / RS. Revista de Ciências Farmacêuticas Básica e Aplicada, v. 26, n. 3, 2005.

GRAPHERY, N. A. Performance-enhancing drugs. The Orthopedic Clinics of North America, v. 26, n. 3, 1995. 
GRUBER, A. J.; POPE JR, H. G. Psychiatric and medical effects of anabolic-androgenic steroid use in women. Psychoterapy and Psychosomatics, v. 69, n. 1, 2000.

HANDELSMAN, D. J.; GUPTA, L. Prevalence and risk factors for anabolic-androgenic steroid abuse in Australian high school students. Internal Journal of Andrology, v. 20, n. 3, 1997.

HILDEBRANDT, T.; LANGENBUCHER, J.; G, S. D. Muscularity concerns among men: development of attitudinal and perceptual measures. Body Image, v. 1, 2004.

IRIART, J. A. B.; ANDRADE, T. M. I. Musculação, uso de esteróides anabolizantes e percepção de risco entre jovens fisiculturistas de um bairro popular de Salvador, Bahia, Brasil. Cad. Saúde Pública, v. 18, p. 1379-87, 2002.

JUNIOR, S.; DA, S. H. A. Morbidade hospitalar por ingestão de esteroides anabólicoandrogênicos (EAA) no Brasil. Revista Brasileira de Medicina do Esporte, v. 19, n. 2, p. 108-111, abr. 2013.

KANAYAMA, G.; COHANE, G. H.; WEISS, R. D.; POPE JR, H. G. Past anabolicandrogenic steroid use among men admitted for substance abuse treatment: an underrecognized problem? The Journal of Clinical Psychiatry, v. 64, n. 2, 2003.

KANAYAMA, G. et al. Body image and attitudes toward male roles in anabolic-androgenic steroid users. The American Journal of Psychiatry, v. 163, n. 4, p. 697-703, abr. 2006.

KANAYAMA, G.; BROWER, K. J.; HUDSON, I., J.; POPE JR., H. G. Treatment of anabolic-androgenic steroid dependence: Emerging evidence and its implications. Drug and Alcohol Dependence, v. 109, 2010.

KUHN, C. M. Anabolic Steroids. Recent progress in Hormone Research, n. 57, 2002.

LENHART, A.; MADDEN, M.; HITLIN, P. Teens and Technology: Youth are leading the transmission to a fuly wired and mobile nation. Pew Internet and American Life Project, 2005.

LISE, M. L. Z.; SILVA, T. S. D. G.; FERIGOLO, M.; BARROS, H. M. T. O abuso de esteróides anabólico-androgênicos em atletismo. Revista da Associação Médica Brasileira, v. 45, n. 4, 1999.

MACEDO, A. R.; OLIVEIRA, C. A. S.; MARANHÃO, É. A.; OKIDA, Y. PARECER CNE/CES 138/2002 HOMOLOGADO. 2002.

MAYCOCK, B.; HOWAT, P. The barriers to illegal anabolic steroid use. Drugs: Education, Prevention and Policy, v. 14, n. 4, 2005.

MIDDLEMAN, A.; DURANT, R. Anabolic steroid use and associated health risk behaviours. Sports Medicine, v. 21, n. 4, 1996.

NILSSON, S.; BAIGI, A.; MARKLUND, B.; FRIDLUND, B. The Prevalence of the use of androgenic anabolic steroids by adolescents in a county of Sweden. European Journal of Public Health, v. 11, n. 2, 2001. 
NOGUEIRA, F. R. DE S.; BRITO, A. DE F.; VIEIRA, T. I.; et al. Prevalência de uso de recursos ergogênicos em praticantes de musculação na cidade de João Pessoa, Paraíba. Revista Brasileira de Ciências do Esporte, v. 37, n. 1, p. 56-64, 2015.

POPE, H. G. J.; KATZ, D. L. Homicide and near-homicide by anabolic steroid users. The Journal of clinical Psychiatry, v. 51, n. 1, 1990.

QUAGLIO, G. et al. Anabolic Steroids: dependence and complications of chronic use. Internal and Emergency Medicine, n. 4, 2009.

SANTOS, A. F.; MENDONÇA, P. M. H.; SANTOS, L. A.; SILVA, N. F.; TAVARES, J. K. L. Anabolizantes: Conceitos segundo praticantes de musculação em Aracaju (SE). Psicologia em Estudo, v. v. 11, n. n. 2, p. 371-380, 2006.

SCOTT, D. M.; WAGNER, J. C.; BARLOW, T. W. Anabolic steroid use among adolescents in Nebraska schools. American Journal of Health-System Pharmacy, v. 53, n. 17, 1996.

SILVA, I. S. M. F.; MOREAU, R. L. M. Uso de esteróides anabólicos androgênicos por praticantes de musculação de grandes academias da cidade de São Paulo. Rev Bras Ciênc Farm, v. 39, p. 327-333, 2003.

SILVA, P. R. P.; MACHADO, L. C.; FIGUEIREDO, C. V.; et al. Prevalência do uso de agentes anabólicos em praticantes de musculação de Porto Alegre. Arq Bras Endocrinol Metab, v. 51, p. 104-10, 2007.

VENâNCIO, D. P.; NÓBREGA, A. C. L. DA; TUFIK, S.; MELLO, M. T. DE. Descriptive assessment on the use of anabolic steroids and their effect on the biochemical and neuroendocrine variables in practitioners of resisted exercise. Revista Brasileira de Medicina do Esporte, v. 16, n. 3, p. 191-195, 2010.

WAGNER, J. C. Abuse of drugs used to enhance athletic performance. American Journal of Hospital Pharmacy, v. 46, n. 10, 1989.

WICHSTROM, L. Predictors of future anabolic androgenic steroid use. Medicine and Science in Sports and Exercise, v. 38, n. 9, 2006.

WU, F. C. Endocrine aspects of anabolic steroids. Clinical Chemistry, v. 43, n. 7, 1997.

Recebido em: 12/04/2018

Revisado em: 21/05/2018

Aprovado em: 28/06/2018

Endereço para correspondência:

wilsonvinicius@gmail.com

Vinicius Dias Wilson

Universidade Federal dos Vales do Jequitinhonha e Mucuri

Rua da Glória, $n^{\circ} 187$

Centro

39100-000 - Diamantina/MG - Brasil. 